\title{
ON A CLASS OF GOLOD HOMOMORPHISMS
}

\author{
EUGENE G@VER and PAOLO SALMON
}

\section{Introduction.}

Properties of the Poincaré series for local rings have been stated in relative form by Levin in [6] and [7] using the concept of a Golod homomorphism. In this paper we show that the natural map

$$
R \rightarrow R_{n}=R / \sum_{i=1}^{n} x_{i} I_{i}
$$

is a Golod homomorphism where $(R, \mathfrak{m})$ is a local ring, $I_{1} \cong \ldots \cong I_{n} \subseteq \mathrm{m}$ are ideals, and $x_{1}, \ldots, x_{n} \in m$ are such that $x_{1}$ is regular on $R$ and each sucessive $x_{i}$ is regular on

$$
R_{i-1}=R / \sum_{j<i} x_{j} I_{j}
$$

This includes generalizations of results obtained by Levin [7], Shamash [8], and Herzog and Steurich [4]. Rings of the form $R_{n}$ were considered by Ghione and Gulliksen in [1] where an explicit formula was given for the Poincare series over $R_{n}$ of a finitely generated $R$-module $M$ such that $I_{n} M=0$. Our result makes use of that formula.

Golod homomorphisms include Golod rings as a special case. If $R$ is a regular local ring and $a$ is an ideal contained in $m^{2}$, then $R / \mathfrak{a}$ is a Golod ring if and only if the natural map $R \rightarrow R / a$ is a Golod homomorphism. Our result shows that for $R$ regular, the rings $R_{n}$ are Golod rings. It follows from [1, theorem 4] that all finite $R_{n}$-modules have rational Poincare series.

In section 1 we fix our notation and review basic facts needed about Golod homomorphisms. The second section establishes two coefficient inequalities for Poincare series. The first inequality, stated in Theorem 2.1, concerns the coefficients of Poincare series defined in the setting of a local homomorphism. The second inequality, stated in Proposition 2.2, relates the coefficients of the Poincare series for the factor ring $R /(I+x J)$ to those of the Poincaré series for $R / I$ and $R / J$ in the case where $I \subseteq J$ and $x$ is regular on $R / I$. An explicit construction is then given for a resolution of $R /(I+x J)$ in terms of resolutions 
for $R / I$ and $R / J$. These results are used in the final section to show that $R \rightarrow R_{n}$ is a Golod homomorphism. In particular, in the special case $I_{1}=\ldots=$ $I_{n}=I \leqq \mathfrak{m}$ with $x_{1}$ regular on $R$ and $x_{i}$ regular $\bmod \left(x_{1}, \ldots, x_{i-1}\right) I$, $R \rightarrow R /\left(x_{1}, \ldots, x_{n}\right) I$ is a Golod homomorphism. When $R$ is regular, $R /\left(x_{1}, \ldots, x_{n}\right) I$ is a Golod ring. These directly generalize a theorem of Levin $[7$, theorem 2.3] and a well-known result of Shamash [8, § 5 corollary 2]. Another example of such a homomorphism is the natural map

$$
R \rightarrow R / \mathfrak{a}_{1}^{s_{1}} \cdots \mathfrak{a}_{n}^{s_{n}}
$$

where the ideals $a_{i}$ are generated by disjoint parts of a regular sequence, a result also obtained by Herzog and Steurich [4, Folgerung 2] using different methods.

\section{Notation, Golod rings and homomorphisms.}

Throughout, $R$ denotes a noetherian local ring with maximal ideal $\mathrm{m}$ and residue field $k=R / \mathrm{m}$. If $M$ is a finitely generated $R$-module, the Betti numbers of $M$ are the integers $b_{i}^{R}(M)=\operatorname{dim}_{k} \operatorname{Tor}_{i}^{R}(k, M)$. The Poincare series of $M$ over the ring $R$ is the formal power series

$$
P_{M}^{R}=\sum_{i=0}^{\infty} b_{i}^{R}(M) z^{i} .
$$

We will use the terms augmented R-algebra and trivial Massey operation as they are defined by Gulliksen in [3] and adopt a corresponding notation. Thus, if the augmented $R$-algebra $F$ has a trivial Massey operation $\gamma$, the function $\gamma$ is defined on some set $C$ of homogeneous cycles in $F$ which represents a minimal set of generators for $\tilde{H}(F)$.

If $f: R \rightarrow R^{\prime}$ is a homomorphism of local rings, we wish to define what it means for $f$ to be a Golod homomorphism. Suppose that $f(\mathfrak{m}) \cong \mathfrak{m}^{\prime}$, that $f$ induces an isomorphism of residue fields $R / \mathrm{m} \approx R^{\prime} / \mathrm{m}^{\prime}=k$, and that $R^{\prime}$ becomes a finite $R$-module via $f$. Let $X$ be a minimal algebra resolution for $k$ over the ring $R$. With these hypotheses, Levin [7] proved

THEOREM 1.1. The following are equivalent:

(a) $P_{k}^{R^{\prime}}=P_{k}^{R} /\left(1-z\left(P_{R^{\prime}}^{R}-1\right)\right)$ and $m^{\prime} H\left(X \otimes_{R} R^{\prime}\right)=0$.

(b) The R'algebra $X \otimes_{R} R^{\prime}$ has a trivial Massey operation and the induced $\operatorname{Tor}^{R}(k, k) \rightarrow \operatorname{Tor}^{R^{\prime}}(k, k)$ is injective.

(c) $X \otimes_{R} R^{\prime}$ has a trivial Massey operation and $\gamma(C) \subset \mathrm{m}^{\prime}\left(X \otimes_{R} R^{\prime}\right)$ where $C$ represents a minimal set of generators for $\tilde{H}\left(X \otimes_{R} R^{\prime}\right)$. 
Definition. A local homomorphism $f: R \rightarrow R^{\prime}$ which satisfies the hypotheses and conditions of Theorem 1.1 is called a Golod homomorphism.

Golod rings can be viewed as a special case.

Definition. A local ring $R$ is a Golod ring if its Koszul complex $K_{R}$ has a trivial Massey operation.

The local ring $R$ is a Golod ring precisely when its m-adic completion $\hat{R}$ is a Golod ring. Since $\hat{R}=S / \mathfrak{a}$ for some regular local ring $(S, \mathfrak{n})$ with $\mathfrak{a} \subseteq \mathfrak{n}^{2}, K_{\hat{R}}$ $=K_{S} \otimes_{S} \hat{R}$. Moreover, the Koszul complex $K_{S}$ is a minimal algebra resolution for $k$ over $S$. It is not hard to verify that when $K_{\hat{R}}$ has a trivial Massey operation, $\gamma(C) \subset \hat{\mathrm{m}} K_{\hat{R}}$ where $C$ represents a minimal set of generators for $H\left(K_{\hat{R}}\right)$. Consequently, $R$ is a Golod ring if and only if the natural map $S \rightarrow S / \mathfrak{a}$ $=\hat{R}$ is a Golod homomorphism.

\section{Coefficient inequalities for Poincaré series.}

Let $f: R \rightarrow R^{\prime}$ be as in the hypotheses of (1.1) and suppose that $M^{\prime}$ is a finitely generated $R^{\prime}$-module.

THeOREM 2.1. If $\mathrm{m}^{\prime} \operatorname{Tor}^{R}\left(k, R^{\prime}\right)=0$ then

$$
P_{M^{\prime}}^{R^{\prime}} \leqq \frac{P_{M^{\prime}}^{R}}{1-z\left(P_{R^{\prime}}^{R}-1\right)}
$$

as an inequality among the coefficients of the Poincare series.

Proof. We must show

$$
b_{n}^{R^{\prime}}\left(M^{\prime}\right)+b_{n-1}^{R^{\prime}}\left(M^{\prime}\right) \leqq \sum_{r=1}^{n} b_{n-r}^{R^{\prime}}\left(M^{\prime}\right) \cdot b_{r-1}^{R}\left(R^{\prime}\right)+b_{n}^{R}\left(M^{\prime}\right)
$$

for $n \geqq 1$ and

$$
b_{0}^{R^{\prime}}\left(M^{\prime}\right) \leqq b_{0}^{R}\left(M^{\prime}\right)
$$

where

$$
P_{M^{\prime}}^{R^{\prime}}=\sum b_{i}^{R^{\prime}}\left(M^{\prime}\right) z^{i}, \quad P_{M^{\prime}}^{R}=\sum b_{i}^{R}\left(M^{\prime}\right) z^{i}, P_{R^{\prime}}^{R}=\sum b_{i}^{R}\left(R^{\prime}\right) z^{i}
$$

The change of rings spectral sequence in the given situation has

$$
E_{p, q}^{2}=\operatorname{Tor}_{p}^{R^{\prime}}\left(\operatorname{Tor}_{q}^{R}\left(k, R^{\prime}\right), M^{\prime}\right) \underset{p}{\Longrightarrow} \operatorname{Tor}_{p+q}^{R}\left(k, M^{\prime}\right) .
$$


By hypothesis, each $R^{\prime}$-module $\operatorname{Tor}_{q}^{R}\left(k, R^{\prime}\right)$ is in fact a $k$-space. Thus $E_{p, q}^{2}$ and all subquotients are finite vector spaces over $k$. We can therefore apply a counting argument similar to one given in $[7,1.3]$ in order to compare the various dimensions over $k$.

Considered over $k, \operatorname{dim} E_{p, q}^{2}=b_{p}^{R^{\prime}}\left(M^{\prime}\right) \cdot b_{q}^{R}\left(R^{\prime}\right) . E_{n, 0}^{r+1}$ is the kernel of $d^{r}: E_{n, 0}^{r}$ $\rightarrow E_{n-r, r-1}^{r}$, and setting $U_{n}^{r}=$ image of $d^{r}$, we have that

$$
\operatorname{dim} E_{n, 0}^{r}=\operatorname{dim} U_{n}^{r}+\operatorname{dim} E_{n, 0}^{r+1}
$$

which upon iteration becomes

$$
\operatorname{dim} E_{n, 0}^{2}=\sum_{r=2}^{n} \operatorname{dim} U_{n}^{r}+\operatorname{dim} E_{n, 0}^{\infty} .
$$

$U_{n}^{r}$ is a subspace of $E_{n-r, r-1}^{r}$ and so

$$
\operatorname{dim} U_{n}^{r} \leqq b_{n-r}^{R^{\prime}}\left(M^{\prime}\right) \cdot b_{r-1}^{R}\left(R^{\prime}\right) .
$$

Since the spectral sequence converges, each $E_{p, q}^{\infty}$ is isomorphic to a subquotient of $\operatorname{Tor}_{p+q}^{R}\left(k, M^{\prime}\right)$ which guarantees that

$$
\operatorname{dim} E_{n, 0}^{\infty} \leqq b_{n}^{R}\left(M^{\prime}\right)
$$

for all $n$. Combining the last three relations,

$$
\operatorname{dim} E_{n, 0}^{2} \leqq \sum_{r=2}^{n} b_{n-r}^{R^{\prime}}\left(M^{\prime}\right) \cdot b_{r-1}^{R}\left(R^{\prime}\right)+b_{n}^{R}\left(M^{\prime}\right)
$$

for $n \geqq 2$. When this is added to

$$
b_{n-1}^{R^{\prime}}\left(M^{\prime}\right) \leqq b_{n-1}^{R^{\prime}}\left(M^{\prime}\right) \cdot b_{0}^{R}\left(R^{\prime}\right)
$$

and we observe that $b_{n}^{R^{\prime}}\left(M^{\prime}\right) \leqq \operatorname{dim} E_{n, 0}^{2}$, inequality (1) is obtained for $n \geqq 2$. Inequality (1) with $n=1$ and inequality (2) both follow from the observation that for $n=0,1$,

$$
b_{n}^{R^{\prime}}\left(M^{\prime}\right) \leqq \operatorname{dim} E_{n, 0}^{2}=\operatorname{dim} E_{n, 0}^{\infty} \leqq b_{n}^{R}\left(M^{\prime}\right) .
$$

The special case of 2.1 with $R$ regular, $R^{\prime}=R / I, I \leqq m^{2}$, and $M^{\prime}=k$ was established by Serre. With those same restrictions, Golod [2] showed that equality is achieved precisely when $K_{R / I}$ has a trivial Massey operation, that is, when $R / I$ is a Golod ring. 2.1 is also an equality when $f: R \rightarrow R^{\prime}$ is a strong Golod homomorphism and $\left(0: \mathrm{m}^{\prime}\right) M^{\prime}=0$ as shown by Levin in $[7$, theorem 4.6].

Proposition 2.2. Let $I \cong J$ be ideals of $R$ and suppose that $x \in \mathfrak{m}$ is regular on R/I. Then 


$$
P_{R /(I+x J)}^{R} \leqq(1+z)\left(P_{R / I}^{R}-1\right)+P_{R / J}^{R}
$$

Proof. Let $p_{i}, m_{i}$, and $n_{i}$ be the respective Betti numbers over $R$ of $R /(I+x J)$, $R / I$, and $R / J$. To establish the inequality, we must show that $p_{1} \leqq m_{1}+n_{1}$ and that $p_{i} \leqq m_{i}+m_{i-1}+n_{i}$ for $i \geqq 2$.

Since $x$ is regular on $R / I$, the inclusion of $J / I$ into $R / I$ followed by multiplication by $x$ yields a monomorphism whose image is $(I+x J) / I$. Thus

$$
0 \rightarrow J / I \rightarrow R / I \rightarrow R /(I+x J) \rightarrow 0
$$

is exact. From the induced homology sequence

$$
\ldots \rightarrow \operatorname{Tor}_{i}^{R}(k, R / I) \rightarrow \operatorname{Tor}_{i}^{R}(k, R /(I+x J)) \rightarrow \operatorname{Tor}_{i-1}^{R}(k, J / I) \rightarrow \ldots
$$

we find that

$$
p_{i} \leqq m_{i}+b_{i-1}^{R}(J / I) .
$$

In a similar manner, exactness of

$$
0 \rightarrow J / I \rightarrow R / I \rightarrow R / J \rightarrow 0
$$

shows that

$$
b_{i-1}^{R}(J / I) \leqq m_{i-1}+n_{i} .
$$

When combined, the two inequalities give the desired relationship for $i \geqq 2$. The inequality for $i=1$ follows after observing that $b_{0}^{R}(J / I) \leqq b_{0}^{R}(J)=b_{1}^{R}(R / J)=n_{1}$.

The inequality of Proposition 2.2 can also be obtained by constructing an $R$ free resolution of $R /(I+x J)$ from resolutions of $R / I$ and $R / J$.

PROPOSITION 2.3. Let $I \subseteq J$ be ideals of $R$ and suppose that $x \in m$ is regular on $R / I$. Let $L$ and $L^{\prime}$ be $R$-free resolutions of $R / I$ and $R / J$ respectively. Then there exists an $R$-free resolution $L^{\prime \prime}$ of $R /(I+x J)$ with $L_{1}^{\prime \prime}=L_{1} \oplus L_{1}^{\prime}$ and $L_{i}^{\prime \prime}$ $=L_{i} \oplus L_{i-1} \oplus L_{i}^{\prime}$ for $i>1$.

Proof. The complex $L^{\prime \prime}$ will turn out to be a two stage mapping cone. First, since $I \cong J$, the identity map on $R=L_{0}=L_{0}^{\prime}$ lifts to a chain map $\psi: L \rightarrow L^{\prime}$. If $f_{I}: L_{1} \rightarrow I$ denotes the boundary map $d_{1}: L_{1} \rightarrow R$ when considered onto its image and if $\tilde{L}$ is the suspension complex of $L$ (i.e., $\tilde{L}_{i}=L_{i+1}$ and $\tilde{d}_{i}=d_{i+1}$ ), then $\left(\tilde{L}, f_{I}\right)$ is an $R$-free resolution of $I$. Let $\tilde{L}^{\prime}$ be defined analogously so as to produce an $R$-free resolution of $J$. Then $\Psi: \tilde{L} \rightarrow \tilde{L}^{\prime}$ with $\tilde{\psi}_{i}=\psi_{i+1}$ is a chain map lifting the inclusion of $I$ into $J$.

The mapping cone $M(\tilde{\psi})$ is acyclic. This can be seen by considering the exact homology sequence 


$$
\ldots \rightarrow H_{i}\left(\tilde{L}^{\prime}\right) \rightarrow H_{i}(M(\tilde{\psi})) \rightarrow H_{i-1}(\tilde{L}) \stackrel{\Psi_{*}}{\rightarrow} H_{i-1}\left(\tilde{L}^{\prime}\right) \rightarrow \ldots
$$

Since $H_{i}\left(\tilde{L}^{\prime}\right)=H_{i+1}\left(L^{\prime}\right)$ and $H_{i-1}(\tilde{L})=H_{i}(L)$ for $i>1, H_{i}(M(\tilde{\psi}))=0$ for $i>1$. When $i=1$, the relevant part of the sequence is

$$
H_{1}\left(\tilde{L}^{\prime}\right)=0 \rightarrow H_{1}(M(\tilde{\psi})) \rightarrow I \subset J
$$

with the right-hand map an inclusion. It follows that $H_{1}(M(\tau))=0$.

The complex $M(\tau)$ has $M_{0}(\tilde{\psi})=L_{1}^{\prime}, M_{i}(\tilde{\psi})=\tilde{L}_{i-1} \oplus \tilde{L}_{i}^{\prime}=L_{i} \oplus L_{i+1}^{\prime}$ for $i>0$, and boundary maps $\delta_{i}$ defined by $\delta_{1}\left(y_{1}, y_{2}^{\prime}\right)=\psi y_{1}+d^{\prime} y_{2}^{\prime}$ and $\delta_{i}\left(y_{i}, y_{i+1}^{\prime}\right)=$ $\left(-d y_{i}, \psi y_{i}+d^{\prime} y_{i+1}^{\prime}\right)$ for $i>1$.

We begin to construct a chain map $\varphi: M(\tilde{\psi}) \rightarrow L$ by first defining $\varphi_{0}$ $=x d_{1}^{\prime}: L_{1}^{\prime} \rightarrow R$ for any fixed $x \in \mathrm{m}$. Since

$$
\varphi_{0} \delta_{1}\left(y_{1}, y_{2}^{\prime}\right)=x d_{1}^{\prime} \psi_{1} y_{1}+x d_{1}^{\prime} d_{2}^{\prime} y_{2}^{\prime}=x d_{1} y_{1},
$$

we may continue the construction by setting $\varphi_{1}\left(y_{1}, y_{2}^{\prime}\right)=x y_{1}$. Indeed, all $\varphi_{i}$ for $i \geqq 1$ can be defined by $\varphi_{i}\left(y_{i}, y_{i+1}^{\prime}\right)=(-1)^{i+1} x y_{i}$. The mapping cone $M(\varphi)$ of this chain map is a complex over $R /(I+x J)$ with

$$
\begin{gathered}
M_{0}(\varphi)=R, M_{1}(\varphi)=L_{1} \oplus M_{0}(\tau)=L_{1} \oplus L_{1}^{\prime}, \text { and } \\
M_{i}(\varphi)=L_{i} \oplus M_{i-1}(\tau)=L_{i} \oplus L_{i-1} \oplus L_{i}^{\prime} \text { for } i>1 .
\end{gathered}
$$

$M(\varphi)$ augmented by the natural map onto $R /(I+x J)$ turns out to be the desired resolution $L^{\prime \prime}$.

Examination of the exact homology sequence for $M(\varphi)$ shows that $H_{i}(M(\varphi))$ $=0$ for $i>1$. The last part of the sequence is

$$
H_{1}(L)=0 \rightarrow H_{1}(M(\varphi)) \rightarrow H_{0}(M(\tau)) \stackrel{\varphi_{*}}{\longrightarrow} H_{0}(L)
$$

with $H_{0}(M(\tau))=L_{1}^{\prime} / \operatorname{Image} \delta_{1}$ and $H_{0}(L)=R / I$. We verify that $\varphi_{*}$ is monic whenever $x$ is regular on $R / I$ by considering $\left[y^{\prime}\right] \in H_{0}(M(\psi))$ such that $\varphi_{*}\left[y^{\prime}\right]$ $=\overline{0} \in R / I$. The representative $y^{\prime} \in L_{1}^{\prime}$ must then have $\varphi_{0} y^{\prime} \in I$. But $\varphi_{0} y^{\prime}=x d_{1}^{\prime} y^{\prime}$ and since $x$ is regular on $R / I, d_{1}^{\prime} y^{\prime} \in I$. Hence there exists $y \in L_{1}$ such that $d_{1} y$ $=d_{1}^{\prime} y^{\prime}$, so

$$
d_{1}^{\prime}\left(y^{\prime}-\psi_{1} y\right)=d_{1}^{\prime} y^{\prime}-d_{1}^{\prime} \psi_{1} y=d_{1}^{\prime} y^{\prime}-d_{1} y=0 .
$$

By exactness, $y^{\prime}-\psi_{1} y=d_{2}^{\prime} y_{2}^{\prime}$ for some $y_{2}^{\prime} \in L_{2}^{\prime}$. Consequently

$$
y^{\prime}=\psi_{1} y+d_{2}^{\prime} y_{2}^{\prime}=\delta_{1}\left(y, y_{2}^{\prime}\right)
$$

which says that $\left[y^{\prime}\right]=[0] \in H_{0}(M(\tau))$. Therefore the map $\varphi_{*}$ in the above exact sequence is monic. It follows that $M(\varphi)$ is acyclic and provides an $R$-free resolution $L^{\prime \prime}$ of $R /(I+x J)$.

Note that by virtue of the mapping cone construction, the boundary maps in 
the complex $L^{\prime \prime}=M(\varphi)$ are completely determined by the boundary maps $d$ and $d^{\prime}$ in the respective complexes $L$ and $L^{\prime}$ and by the chain map $\psi: L \rightarrow L^{\prime}$ lifting $1_{R}$. Specifically,

$$
\begin{aligned}
d_{1}^{\prime \prime}: & L_{1} \oplus L_{1}^{\prime} \rightarrow R \text { is defined by } d_{1}^{\prime \prime}\left(y_{1}, y_{1}^{\prime}\right)=d y_{1}+x d^{\prime} y_{1}^{\prime}, \\
d_{2}^{\prime \prime}: & L_{2} \oplus L_{1} \oplus L_{2}^{\prime} \rightarrow L_{1} \oplus L_{1}^{\prime} \text { is defined by } \\
& d_{2}^{\prime \prime}\left(y_{2}, y_{1}, y_{2}^{\prime}\right)=\left(d_{2} y_{2}+x y_{1},-\psi y_{1}-d^{\prime} y_{2}^{\prime}\right), \text { and } \\
d_{i}^{\prime \prime}: & L_{i} \oplus L_{i-1} \oplus L_{i}^{\prime} \rightarrow L_{i-1} \oplus L_{i-2} \oplus L_{i-1}^{\prime} \text { for } i \geqq 3 \text { is } \\
& \text { defined by } d_{i}^{\prime \prime}\left(y_{i}, y_{i-1}, y_{i}^{\prime}\right)= \\
& \left(d_{i} y_{i}+(-1)^{i} x y_{i-1}, d y_{i-1},-\psi y_{i-1}-d^{\prime} y_{i}^{\prime}\right) .
\end{aligned}
$$

If bases are picked for the free modules and if the boundary maps are defined by matrices with respect to those bases, then setting

$$
A^{(i)}=\left[d_{i}\right], B^{(i)}=\left[d_{i}^{\prime}\right], C^{(i)}=\left[\psi_{i}\right], D^{(i)}=\left[d_{i}^{\prime \prime}\right],
$$

and $E(i)=$ an identity matrix of the appropriate size, we have

$$
\begin{gathered}
D^{(1)}=\left[\begin{array}{lll}
A^{(1)} & x B^{(1)}
\end{array}\right], \quad D^{(2)}=\left[\begin{array}{ccc}
A^{(2)} & x E^{(2)} & 0 \\
0 & -C^{(1)} & -B^{(2)}
\end{array}\right], \\
\text { and } D^{(i)}=\left[\begin{array}{ccc}
A^{(i)} & (-1)^{i} x E^{(i)} & 0 \\
0 & A^{(i-1)} & 0 \\
0 & -C^{(i-1)} & -B^{(i)}
\end{array}\right] \quad \text { for } i \geqq 3
\end{gathered}
$$

Proposition 2.2 clearly follows from 2.3 . The resolution $L^{\prime \prime}$ need not be minimal, however, even when both $L$ and $L^{\prime}$ are minimal. $L^{\prime \prime}$ is minimal in particular cases. For example, if $I=\sum_{j<i} x_{j} I_{j}$ and $J=I_{i}$ where $x_{1}, \ldots, x_{i}$ and $I_{1}, \ldots, I_{i}$ satisfy the hypotheses of the next theorem 3.1 and $L, L^{\prime}$ are minimal resolutions, then $L^{\prime \prime}$ is a minimal resolution of

$$
R /(I+x J)=R / \sum_{j=1}^{i} x_{j} I_{j}
$$

as a consequence of 2.2 and 3.1 .

\section{The Golod homomorphism $R \rightarrow R_{n}$.}

THEOREM 3.1. Let $(R, m)$ be a local ring with ideals $I_{1} \subseteq \ldots \subseteq I_{n} \subseteq m$ and suppose that $x_{1}, \ldots, x_{n} \in m$ satisfy: $x_{1}$ is regular on $R$ and for each $i>1, x_{i}$ is regular on $R_{i-1}=R / \sum_{j<i} x_{j} I_{j}$. Let $M$ be a finitely generated $R$-module such that $I_{n} M=0$. Then 


$$
P_{M}^{R_{n}}=\frac{P_{M}^{R}}{1-z\left(P_{R_{n}}^{R}-1\right)} .
$$

In particular, the natural map $R \rightarrow R_{n}$ is a Golod homomorphism.

Proof. With the given hypotheses, Corollary 6 of [1] applies and shows that

$$
P_{M}^{R_{n}}=\frac{P_{M}^{R}}{f_{n}(z)}
$$

where for $1 \leqq i \leqq n$,

$$
\begin{aligned}
f_{i}(z) & =(1+z)^{i}-z \sum_{j<i}(1+z)^{j} P_{R / I_{i-j}}^{R} \\
& =(1+z) f_{i-1}(z)-z P_{R / I_{i}}^{R} .
\end{aligned}
$$

Theorem 2.1 shows that

$$
\frac{P_{M}^{R}}{f_{n}(z)} \leqq \frac{P_{M}^{R}}{1-z\left(P_{R_{n}}^{R}-1\right)}
$$

If we establish

$$
P_{R_{n}}^{R}-1 \leqq \frac{1-f_{n}(z)}{z},
$$

then the reverse inequality of (2) will also hold in which case equations (1) and (2) will establish the desired equation for the Poincare series.

Inequality (3) is obtained by induction on $n$. When $n=1$,

$$
P_{R_{1}}^{R}-1=P_{R / x_{1} I_{1}}^{R}-1=P_{R / I_{1}}^{R}-1=\frac{1-f_{1}(z)}{z} .
$$

Assume that for $n-1 \geqq 1$,

$$
P_{R_{n-1}}^{R}-1 \leqq \frac{1-f_{n-1}(z)}{z}
$$

From Proposition 2.2,

$$
P_{R_{n}}^{R}=P_{R /\left(\sum_{j<n} x_{j} I_{j}+x_{n} I_{n}\right)}^{R} \leqq(1+z)\left(P_{R_{n-1}}^{R}-1\right)+P_{R / I_{n}}^{R} .
$$

When combined with $(4)$ this gives

$$
\frac{P_{R_{n}}^{R}-P_{R / I_{n}}^{R}}{(1+z)} \leqq P_{R_{n-1}}^{R}-1 \leqq \frac{1-f_{n-1}(z)}{z}
$$


Hence

$$
P_{R_{n}}^{R} \leqq \frac{(1+z)\left(1-f_{n-1}(z)\right)+z P_{R / I_{n}}^{R}}{z}
$$

from which (3) readily follows.

To show that $R \rightarrow R_{n}$ is a Golod homomorphism we verify (a) of 1.1. With $M=k, I_{n} k=0$; the above argument then establishes the appropriate formula for the Poincare series. The remaining condition of 1.1 (a) holds since $R_{n}$ is a factor ring of $R$ from which it follows that an $R_{n}$-module is a $k$-space whenever it is a $k$-space as an $R$-module.

An immediate consequence of 3.1 and $[1$, theorem 4$]$ is

COROllaRY 3.2. If $R$ is regular, $R_{n}$ is a Golod ring. If $M$ is a finite $R_{n}$-module, $P_{M}^{R_{n}}$ is rational.

With $n=1$ in 3.1, $R \rightarrow R / x I$ is a Golod homomorphism. This is Levin's [7, theorem 2.3] for the case $I \subseteq \mathfrak{m}$ and $x \in \mathfrak{m}$ a regular element. When $n=1$ in 3.2 with $R$ regular and $x \neq 0, R / x I$ is a Golod ring. This is a result of Shamash $[8, \S$ 5 cor. 2]. Both of these results now generalize to the case $R /\left(x_{1}, \ldots, x_{n}\right) I$ with $x_{1}$ regular and $x_{i}$ regular mod $\left(x_{1}, \ldots, x_{i-1}\right) I$ by taking $I_{1}=\ldots=I_{n}=I \cong \mathfrak{m}$. Other interesting examples of rings of the form $R_{n}$ come from

COROllary 3.3. Let $(R, \mathfrak{m})$ be a local ring with ideals $\mathfrak{a}_{1}, \mathfrak{a}_{2}, \ldots, \mathfrak{a}_{n}$ generated by disjoint parts of a regular sequence. Suppose that $n>1$ or the integer $s_{1}>1$. Then the natural map

$$
R \rightarrow R / \mathfrak{a}_{1}^{s_{1}} \cdots \mathfrak{a}_{n}^{s_{n}}
$$

is a Golod homomorphism.

Proof. We may write

$$
\mathfrak{a}_{1}=\left(x_{11}, \ldots, x_{1 t_{1}}\right), \ldots, \mathfrak{a}_{n}=\left(x_{n 1}, \ldots, x_{n t_{n}}\right)
$$

where $x_{11}, \ldots, x_{1 t_{1}}, \ldots \ldots, x_{n t_{n}}$ constitutes a regular sequence. Let $\mathfrak{a}$ $=\mathfrak{a}_{1}^{s_{1}} \cdots \mathfrak{a}_{n}^{s_{n}}$. If $n=1$, set $J=R$; otherwise, set $J=\mathfrak{a}_{2}^{s_{2}} \cdots \mathfrak{a}_{n}^{s_{n}}$. In either case we have

$$
\mathfrak{a}=x_{11}\left(x_{11}\right)^{s_{1}-1} J+x_{12}\left(x_{11}, x_{12}\right)^{s_{1}-1} J+\ldots+x_{1 n}\left(x_{11}, \ldots, x_{1 n}\right)^{s_{1}-1} J .
$$

If $I_{i}=\left(x_{11}, \ldots, x_{1 i}\right)^{s_{1}-1} J$, then $I_{1} \subseteq \ldots \subseteq I_{n} \subset \mathrm{m}$ and $\mathrm{a}=\sum_{i=1}^{n} x_{1 i} I_{i}$. Observe that $\sum_{j<i} x_{1 j} I_{j}$ is an ideal generated by monomials in $x_{11}, \ldots, x_{1 i-1}$ and $x_{21}, \ldots, x_{n t_{n}}$ which is a part of the regular sequence disjoint from $x_{1 i}$. It therefore follows 
from [5, lemma 3] that $x_{1 i}$ is regular on $R / \sum_{j<i} x_{1 j} I_{j}$ and so the natural map $R$ $\rightarrow R / a$ is a Golod homomorphism by 3.1 .

This corollary has been obtained independently by Herzog and Steurich $[4$, Folgerung 2]. Several examples found in the literature follow from it. For instance, $R \rightarrow R /\left(x_{1}, \ldots, x_{n}\right)^{s}$ for $s>1$ is a Golod homomorphism where $x_{1}, \ldots, x_{n}$ is a regular sequence. In particular for a field $K$,

$$
K\left[\left[X_{1}, \ldots, X_{n}\right]\right] /\left(X_{1}, \ldots, X_{n}\right)^{s}
$$

with $s>1$ is a Golod ring (cf. [2]). Rings of the form

$$
K\left[\left[X_{1}, \ldots, X_{4}\right]\right] /\left(X_{1}, X_{2}\right)^{i}\left(X_{3}, X_{4}\right)^{j}
$$

are also Golod. The simplest example of a ring satisfying the hypotheses of Theorem 3.1 but not of the type considered in 3.3 is

$$
\begin{array}{r}
R_{n}=K[[X, Y, Z]] /(X, Y)(X, Z) \\
\left(R=K[[X, Y, Z]], x_{1}=X, x_{2}=Y \text {, and } I_{1}=I_{2}=(X, Z)\right) .
\end{array}
$$

\section{REFERENCES}

1. F. Ghione and T. H. Gulliksen, Some reduction formulas for the Poincaré series of modules, Atti Accad. Naz. Lincei Rend. Cl. Sci. Fis. Mat. Natur. (8) 58 (1975), no. 2, 82-91.

2. E. S. Golod. On the homology of some local rings, Soviet Math. Dokl. 3 (1962), 745-748.

3. T. H. Gulliksen, Massey operations and the Poincaré series of certain local rings, J. Algebra 22 (1972), 223-232.

4. J. Herzog and M. Steurich, Golodideale der Gestalt a $\cap$ b, J. Algebra 58 (1979), 31-36.

5. I. Kaplansky, R-sequences and homological dimension, Nagoya Math. J. 20 (1962), 195-199.

6. G. Levin, Local rings and Golod homomorphisms, J. Algebra 37 (1975), 266-289.

7. G. Levin, Lectures on Golod homomorhisms, Matematiska institutionen, Stockholms universitet, No. 15, 1976.

8. J. Shamash, The Poincaré series of a local ring, J. Algebra 12 (1969), 453-470.

DEPARTMENT OF MATHEMATICS NORTHEASTERN UNIVERSITY BOSTON, MASS. 02115 U.S.A.
ISTITUTO MATEMATICO UNIVERSITẢ DI GENOVA 16132 GENOVA ITALY 